\title{
Aplicación de isótopos estables como indicadores de flujos de energía en ambientes costeros de Uruguay
}

\section{Application of stable isotopes as indicators of energy fluxes in coastal environments of Uruguay}

Bergamino, Leandro (1); Tudurí, Adriana (2); Bueno, Carolina (2); Brugnoli, Ernesto (2); Valenzuela, Luciano (3-4); Martínez, Ana (5); Perez Becoña, Laura (1); Scarabino, Fabrizio (1); García-Rodríguez, Felipe (1).

(1) Centro Universitario Regional Este (CURE), Universidad de la República (UdelaR), Rocha, Uruguay.

(2) Universidad de la República, Facultad de Ciencias, IECA, Oceanografía y Ecología Marina Uruguay,

(3) Consejo Nacional de Investigaciones Científicas y Técnicas (CONICET), Laboratorio de Ecología Evolutiva Humana (LEEH, Núcleo de Estudios Interdisciplinarios sobre Poblaciones Humanas de Patagonia Austral (NEIPHPA), Universidad Nacional del Centro de la Provincia de Buenos Aires, Unidad de Enseñanza Universitaria Quequén, Buenos Aires, Argentina.

(4) Department of Biology, University of Utah, Salt Lake City, Utah, EE.UU.

(5) Unidad de Gestión Pesquera Atlántica, Dirección Nacional de Recursos Acuáticos (DINARA), Rocha, Uruguay.

Contacto: lbergamino@gmail.com

RECIBIDO: 22/3/2017 - APROBADO: 6/6/2017

\section{Resumen}

El análisis de isótopos estables en sedimentos, vegetales y animales representa una herramienta de importancia para estudios ecológicos, reconstrucciones paleoclimáticas y paleoambientales. Con base en la diferenciación isotópica entre productores primarios, esta técnica ha tenido un gran impacto en la identificación de flujos de energía entre ecosistemas terrestres y acuáticos adyacentes y en la estructura trófica. Se sintetizan aquí la nomenclatura y los principios básicos para la aplicación de isótopos estables en estudios de ambientes acuáticos. Además, se muestra su utilidad describiendo tres ejemplos recientes en ambientes costeros de Uruguay con diferentes objetivos: 1) evaluar el origen de la materia orgánica en sedimentos del Río de la Plata, 2) determinar la importancia trófica de una especie de diatomea en playas arenosas de Uruguay, y 3) evaluar la influencia de la materia orgánica antropogénica en la Bahía de Montevideo. La composición isotópica de las fuentes de materia orgánica permitió inferir los mecanismos involucrados en la transferencia de materia orgánica en los ecosistemas costeros. En esta revisión se subrayan las ventajas de este marcador isotópico de carbono que permite discriminar fuentes de materia orgánica. Asimismo, la combinación con otros análisis complementarios como la espectrofluorometría o los biopolímeros resulta importante en investigaciones de funcionamiento ecosistémico.

Palabras clave: Carbono, nitrógeno, relación c/n, fuentes de materia orgánica, estuarios.

\begin{abstract}
Stable carbon and nitrogen isotope composition of plants, sediments and animals has been used as a common tool employed in different studies including ecology, and paleoenvironmental reconstruction. Because of the different isotopic values among primary producers, this is a powerful tool to trace pathways of exchange of organic matter between ecosystems. We describe the basic principles, including nomenclature, of the application the isotopic approach in coastal environmental studies. Furthermore, we present three recent studies with the isotopic approach for: 1) assess the distribution of deposited organic matter within the Río de la Plata estuary, 2) determine the trophic importance of a diatom from a surf zone in sandy beach food webs, and 3) assess the influence of the anthropogenic organic matter within the Montevideo Bay. The isotopic composition of organic matter sources allowed the study of different aspect of ecosystem function including the characterization of the organic matter pathways. This review highlighted the significant utility of this complementary tool for the study of ecological process and could be useful in the identification of changes in coastal environments.

Keywords: Carbon, nitrogen, $\mathrm{C} / \mathrm{N}$ ratio, organic matter sources, estuaries.
\end{abstract}




\section{Tabla de contenidos}

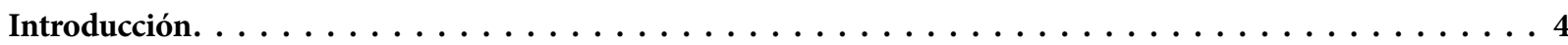

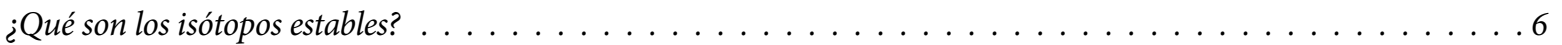

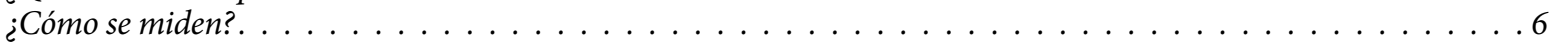

¿Cómo se generan variaciones en la distribución de isótopos? . . . . . . . . . . . . . . . . . . . . . . . . . . . . . . . .

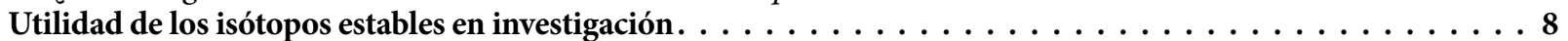

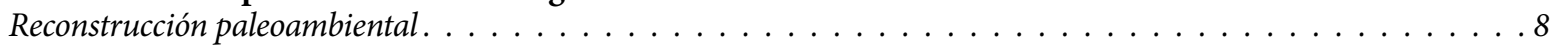

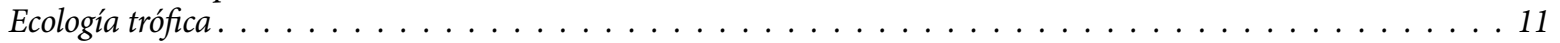

Indicadores de impacto humano . . . . . . . . . . . . . . . . . . . . . . . . . . 13

Casos de estudio . . . . . . . . . . . . . . . . . . . . . . . . . . . . . . . . . . . .

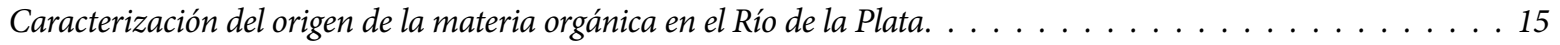

Cambios en las relaciones alimentarias en playas arenosas . . . . . . . . . . . . . . . . . . . . . 18

Materia orgánica antropogénica en los sedimentos de la Bahía de Montevideo . . . . . . . . . . . . . . . . . . 21

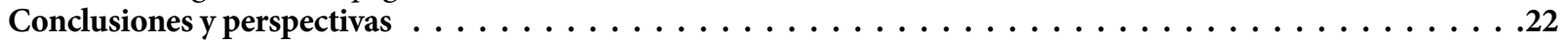

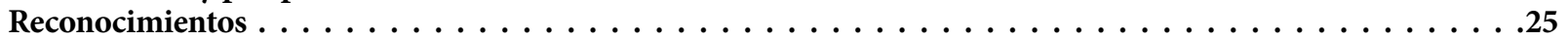

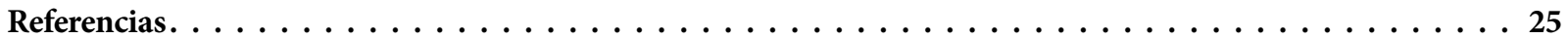

\section{Introducción}

Desde la década de 1970 el uso del análisis de isótopos estables sobre materiales biológicos en estudios ambientales ha crecido exponencialmente. Los desarrollos tempranos de esta herramienta durante la década de 1970 y principios de 1980 se caracterizaron por diferenciar isotópicamente entre las vías fotosintéticas de plantas C3 y C4 (O’Leary, 1981) y, a su vez, por distinguir a aquellos animales actuales o extintos que se alimentaron de ellas (Cerling, et al., 1999).

El siguiente desarrollo importante en los estudios de redes tróficas fue el uso de isótopos estables de nitrógeno como medidores directos de la posición trófica de los consumidores, que permitió establecer conexiones tróficas y cuantificaciones de dieta (década de 1980 y 1990; Martínez del Río, et al., 2009). A fines de la década de 1980 y durante toda la década de 1990 se generó un gran interés en el uso de isótopos estables en estudios para discernir patrones migratorios y de movimiento en animales, analizando las variaciones geográficas en los isótopos estables de distintos elementos ( $\mathrm{C}$ y $\mathrm{H}$ especialmente) (Rubenstein y Hobson, 2004). En la última década, los avances tecnológicos y las propuestas interdisciplinarias han revolucionado ciertas áreas antes no trabajadas, incorporando análisis de isótopos estables de compuestos específicos, como, por ejemplo, las reconstrucciones paleoclimáticas a través de la medición de isótopos de $\mathrm{H}$ en derivados de ceras vegetales extraídos de testigos en lagos u océanos (Berke, et al., 2012). Asimismo, en los últimos años han emergido herramientas estadísticas que permiten realizar aproximaciones cuantitativas para el análisis de isótopos estables, que contemplan la incertidumbre en los valores de entrada y que han llevado a un acelerado uso de los isótopos estables (Phillips, et al., 2014; Craven, et al., 2017).

Mas allá de estos desarrollos, en muchas regiones los estudios isotópicos aún pueden ayudar a llenar un gran vacío de información que sería muy difícil conseguir de otra manera, por ejemplo, la caracterización ambiental y paleoambiental de la costa de Uruguay. La obtención de bases de datos isotópicos locales para nuestra región, incluyendo fauna, flora y sedimentos, en diferentes ambientes presenta un desafío actual: cómo evaluar la circulación de isótopos para permitir caracterizar ciclos biogeoquímicos y describir redes tróficas.
Este trabajo destaca el potencial, importancia y aportes de los isótopos estables al conocimiento de la estructura y funcionamiento de los ecosistemas costeros en Uruguay. Para ello se describen tres casos de estudio que involucran sitios costeros de Uruguay en los que se analiza: 1) la importancia de determinar el origen de la materia orgánica a lo largo de un gradiente salino en un estuario (Bergamino, et al., 2017), 2) la caracterización de las redes tróficas en ambiente de playas (Bergamino, et al., 2016), y 3 ) la evaluación de la influencia humana en una zona altamente urbanizada (Bueno, 2016).

\section{¿Qué son los isótopos estables?}

Los isótopos son formas diferentes de un mismo elemento químico que difieren en el número de neutrones presentes en su núcleo y, por lo tanto, en su masa (Hoefs, 2009). Estas formas isotópicas pueden dividirse en dos tipos: estables e inestables (radioactivos). En este trabajo no se tratará la temática de isótopos en general sino de isótopos estables. El carbono (C) presenta dos formas estables: el liviano ${ }^{12} \mathrm{C}$, con 6 protones y 6 neutrones y con una masa atómica de 12 , y el pesado ${ }^{13} \mathrm{C}$, que presenta 6 protones y 7 neutrones en su núcleo. Los isótopos ocurren naturalmente en los sistemas, participan en todos los procesos geoquímicos de la Tierra y las formas más livianas de un elemento ocurren en mayor proporción que los pesados. La estabilidad de los isótopos se caracteriza por un número de protones y neutrones similares que permite mantener bajas las fuerzas de repulsión de los protones cargados positivamente sin sufrir un decaimiento radioactivo (Hoefs, 2009).

\section{¿Cómo se miden?}

La abundancia natural isotópica se expresa en una escala delta $(\delta)$ que representa una desviación (en \%o) de la composición isotópica de una muestra respecto a un estándar internacional aceptado y determinado por la siguiente ecuación:

$$
\delta \mathrm{X}=\left(R_{\text {muestra }}-R_{\text {standard }}\right) / R_{\text {standard }}{ }^{\star} 1000
$$

donde $\mathrm{X}$ es el elemento en su forma más pesada y $\mathrm{R}$ es la razón del isótopo pesado sobre el liviano (las relaciones más usadas incluyen: ${ }^{2} \mathrm{H} /{ }^{1} \mathrm{H},{ }^{13} \mathrm{C} /{ }^{12} \mathrm{C},{ }^{15} \mathrm{~N} /{ }^{14} \mathrm{~N}$ y $\left.{ }^{18} \mathrm{O} /{ }^{16} \mathrm{O}\right)$. Debido 
a que las relaciones de las abundancias naturales son muy pequeñas, este tipo de expresión de valores isotópicos relativos a un estándar y multiplicado por 1000 permite mostrar estas pequeñas diferencias fraccionarias de una forma conveniente.

Las relaciones isotópicas se miden con un espectrómetro de masas que separa iones y moléculas de acuerdo a su masa. Este instrumento está basado en el diseño original de Nier (1947) que consiste en un sistema de entrada de la muestra, una fuente para ionizar las moléculas, un campo magnético para detectar y separar las partículas cargadas en base a su relación masa/carga, un sistema de trampa de iones, y un detector. En un espectrómetro de masas las moléculas orgánicas son introducidas en forma de gas, frecuentemente como $\mathrm{H}_{2}$, $\mathrm{CO}_{2}, \mathrm{~N}_{2}, \mathrm{y} \mathrm{SO}_{2}$, y la ionización del gas es conseguida mediante el bombardeo de electrones. Los aspectos relacionados a la preparación de muestras luego de su colecta se detallan en Teece y Fogel (2004). Muy brevemente, las muestras son usualmente secadas en estufa, molidas usando mortero de ágata y finalmente pesadas en cápsulas de estaño (que suelen tener un tamaño de $8 \mathrm{~mm}$ de alto por $5 \mathrm{~mm}$ de diámetro). El bajo costo de los análisis isotópicos junto con el escaso material necesario para analizar en la muestra hace de esta aproximación una alternativa muy ventajosa para estudios de diferentes disciplinas, entre ellas, la ecología, la arqueología, la geología y la fisiología. Muestras de diferente tipo necesitarán cantidades diferentes para analizar. Por ejemplo, para las muestras de músculo en peces y de la mayoría de invertebrados es necesario obtener $1 \mathrm{mg}$, mientras que para material de plantas vasculares se necesita pesar alrededor de $3 \mathrm{mg}$ dependiendo de la relación $\mathrm{C} / \mathrm{N}$.

\section{¿Cómo se generan variaciones en la distribución de isótopos?}

La utilidad de los isótopos estables en estudios (paleo) ecológicos y (paleo) ambientales se basa en que diferentes factores y procesos físicos (como por ejemplo evaporación y difusión), químicos y biológicos o enzimáticos generan patrones de distribución en la proporción del isótopo pesado sobre el liviano en ambientes naturales, así como en animales, plantas y productos hechos por el hombre. El término usado para hacer referencia a este cambio isotópico es el fraccionamiento y se denota con el símbolo $\Delta$ como la diferencia isotópica entre los dos valores $(\Delta \mathrm{a}-\mathrm{b}=\delta \mathrm{a}-\delta \mathrm{b})($ Sulzman, 2007).

Existen diferentes formas de fraccionamiento, aunque en este trabajo solo se consideran las dos formas más comunes consignadas en la literatura relacionada a ecología de isótopos livianos. En el fraccionamiento que ocurre durante las reacciones cinéticas (fraccionamiento cinético) las moléculas que contienen los isótopos livianos reaccionan generalmente más rápido que las moléculas que contienen el isótopo pesado. Este fenómeno producirá una abundancia relativa mayor de isótopos livianos, y en el sustrato remanente una abundancia menor de los isótopos livianos (por lo tanto, una abundancia relativa mayor de los isótopos pesados). Este fraccionamiento es promovido por reacciones no reversibles e incompletas, es decir, no todo el substrato es consumido. La mayoría de reacciones bioquímicas (por ejemplo, fotosíntesis y procesos bacterianos) se enmarcan en este tipo de fraccionamiento (Hoefs, 2009). Otro tipo de fraccionamiento ocurre en reacciones de equilibrio, donde los isótopos más pesados tienden a acumularse cuando las uniones son más fuertes. Uno de los ejemplos más claros de este tipo de fraccionamiento es la concentración de los isotopos más pesados del agua $\left({ }^{18} \mathrm{O} y^{2} \mathrm{H}\right)$ en la fase líquida con respecto al vapor de agua.

\section{Utilidad de los isótopos estables en investigación}

\section{Reconstrucción paleoambiental}

Una de las aplicaciones más comunes de los isótopos estables es su uso como registros ambientales de moléculas bióticas y abióticas, que permite la reconstrucción de procesos ambientales (West, et al., 2006). En particular, los valores de $\delta^{13} \mathrm{C}$ son utilizados para identificar el origen de la materia orgánica, ya que los valores isotópicos en las plantas son función de la vía de fotosíntesis $\mathrm{C} 3$ o C4, y sus valores son escasamente afectados por los procesos de diagénesis bioquímica acuática y terrestre en comparación con otros isótopos como Ny S (Michener y Schell, 1994; Rosenbauer, et al., 2009). En este contexto, las plantas terrestres que se caracterizan por tener vías fotosintéticas $\mathrm{C} 3$ muestran valores de $\delta^{13} \mathrm{C}$ con un rango entre -30 y $-21 \%$. A su vez, plantas con vías fotosintéticas $C 4$ tienen valores típicos de $\delta^{13} \mathrm{C}$ entre -17 y-9\%o debido a que la enzima RUBISCO actúa en un ambiente relativamente cerrado y, en consecuencia, utiliza $\mathrm{CO}_{2}$ con el isótopo pesado ${ }^{13} \mathrm{C}$, que no es liberado a la atmósfera como sucede con las plantas C3 (Gannes, et al., 1998). Por su parte, el carbono orgánico particulado marino presenta valores típicos de $\delta^{13} \mathrm{C}$ que van desde -24 a -18\%o (Meyers, 1994; Lamb, et al., 2006; Rosenbauer, et al., 2009). Las algas de agua dulce en sistemas dominados por vegetación C3 tienden a presentar valores de $\delta^{13} \mathrm{C}$ entre -30 y $-25 \%$. Estas diferencias isotópicas entre productores primarios son explicadas principalmente por una utilización diferencial de $\mathrm{HCO}_{3}{ }^{-}$y $\mathrm{CO}_{2}$ disuelto que presentan valores isotópicos diferentes, con mayores valores de $\delta^{13} \mathrm{C}$ en $\mathrm{HCO}_{3}{ }^{-}$que en $\mathrm{CO}_{2}$ (Lamb, et al., 2006). Debido a las condiciones de $\mathrm{pH}$, en el ambiente marino existe menor disponibilidad de $\mathrm{CO}_{2}$, lo que provoca una mayor utilización de $\mathrm{HCO}_{3}-$ por el fitoplancton y, en consecuencia, mayores valores de $\delta^{13} \mathrm{C}$ en comparación con el fitoplancton de agua dulce. Además, existen otros factores que generan variaciones espacio-temporales de $\delta^{13} \mathrm{C}$ en los productores primarios de sistemas acuáticos incluyendo la clorofila $a$ (Gu, et al., 1996), la intensidad de luz y la velocidad del flujo de agua (Finlay, et al., 1999), que permiten un alto nivel de entendimiento sobre flujos de energía entre los componentes de un ecosistema.

Las relaciones de carbono orgánico/nitrógeno total $(\mathrm{C} / \mathrm{N})$ han sido útiles en combinación con valores de $\delta^{13} \mathrm{C}$ en reconstrucciones de paleoambientales y en secuencias de sedimentos (Lamb, et al., 2006; Perez, et al., 2017). Las plantas vasculares tienen valores altos de la relación $\mathrm{C} / \mathrm{N}$ usualmente mayores a 12 debido a la abundancia de celulosa y lignina, mientras que el fitoplancton marino se hace más rico en $\mathrm{N}$ y los valores de la relación C/N varían entre 5 y 7 (Meyers, 1994; Lamb, et al., 2006). Esto permite emplearlos como marcadores ambientales para interpretar el origen de la materia orgánica mediante un gráfico bidimensional entre la relación $\mathrm{C} / \mathrm{Ny} \delta^{13} \mathrm{C}$ que distingue fuentes terrestres y/o marinas (Figura 1). De esta manera, la identificación de cambios de estado en un sistema incluyendo el tipo de vegetación dominante (C3 vs C4 o terrestres vs acuáticas) ha permitido inferir cambios ambientales relacionados a las variaciones del nivel del mar, cambios de salinidad y de procesos climáticos. Por ejemplo, mediante el estudio isotópico 


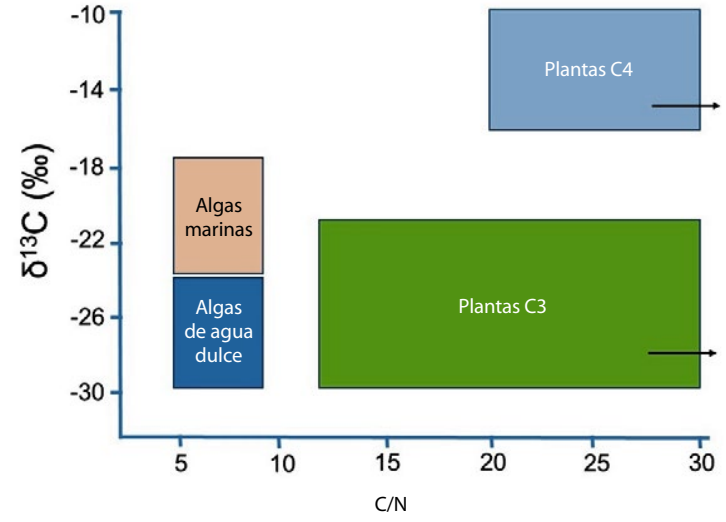

Figura 1. Rango de variación en $\delta^{13} \mathrm{C}$ y $\mathrm{C} / \mathrm{N}$ para diferentes componentes de ambientes costeros. Los datos fueron tomados de Meyers (1994), Lamb et al. (2006) y sus referencias.

de testigos de sedimento se pueden distinguir cambios en los patrones de descarga de agua dulce, ya que estos afectan los patrones de salinidad y, en consecuencia, el tipo de vegetación dominante (Byrne, et al., 2001; Bergamino, et al., 2017).

\section{Ecología trófica}

Otra aplicación de isótopos estables de carbono y nitrógeno ampliamente usada incluye la investigación en ecología para la descripción de las relaciones tróficas, identificación de cambios en la dieta de organismos, la evaluación de áreas geográficas de alimentación y los patrones de movimiento migratorios en animales (Rubenstein y Hobson, 2004; Rodríguez-Graña, et al., 2008; Martínez del Río, et al., 2009; Franco-Trecu, et al., 2014). Estas inferencias tróficas se basan en que la composición isotópica de $\delta^{13} \mathrm{C}$ en los tejidos de los organismos es determinada principalmente por la composición isotópica de las fuentes de alimentos (Vander Zanden y Rasmussen, 2001; McCutchan, et al., 2003; Figura 2). El fraccionamiento de $\delta^{13} \mathrm{C}$ en animales ocurre durante

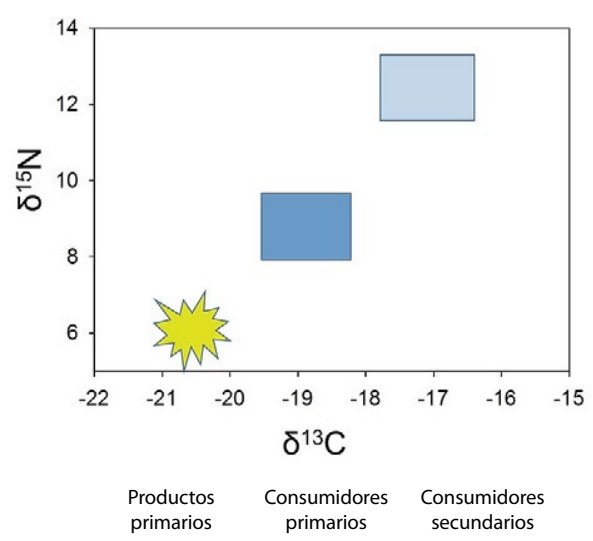

Figura 2. Diagrama conceptual del enriquecimiento trófico en $\delta^{13} \mathrm{C}$ y $\delta^{15} \mathrm{~N}(\%)$ desde los productores primarios, consumidores primarios y secundarios para un sistema costero. Los rangos de variación isotópicos en los componentes ecosistémicos fueron adaptados de Bergamino et al. (2016) y de Rodríguez-Graña et al. (2008).

el proceso de respiración mediante la utilización preferente del $\mathrm{CO}_{2}$ formado con el isótopo liviano ${ }^{12} \mathrm{C}$ (DeNiro y Epstein, 1978). Sin embargo, este proceso determina un escaso enriquecimiento trófico de $\delta^{13} \mathrm{C}$ entre los tejidos del consumidor y su fuente de C ( 0-1\%o), permitiendo así el uso de $\delta^{13} \mathrm{C}$ para identificar la principal fuente de dieta en la base de un ecosistema. A su vez, los valores de $\delta^{15} \mathrm{~N}$ muestran un enriquecimiento en los tejidos de un organismo respecto a su dieta de entre $2-4 \%$, debido principalmente al fraccionamiento por procesos de desaminación y transaminación, que resultan en la eliminación del isótopo liviano ${ }^{14} \mathrm{~N}$ como producto de excreción (Gannes, et al., 1998). Por esta razón, los valores de $\delta^{15} \mathrm{~N}$ posibilitan la estimación de la posición trófica de un organismo (Post, 2002). Este enriquecimiento trófico de $\delta^{15} \mathrm{~N}$ puede ser variable entre grupos de animales y puede estar relacionado con el tipo de dieta (McCutchan, et al., 2003; Vanderklift y Ponsard, 2003; Caut, et al., 2009).

\begin{tabular}{|l|c|c|}
\hline \multirow{2}{*}{ Escala temporal } & \multicolumn{2}{|c|}{ Tipo de tejido } \\
\cline { 2 - 3 } Horas & Renovable & Crecimiento continuo \\
\hline Días & Contenido estomacal & \\
Semanas & Hígado & \\
Entre 1 y 6 meses & Músculo, células sanguíneas, pelo, plumas, piel & \\
Anual (series temporales) & Pelo, plumas & Barbas de ballena, conchas de moluscos, \\
uñas, dientes
\end{tabular}

Tabla 1. Tiempo estimado que integran diferentes tipos de tejido sobre información dietaria en un organismo. Se distinguen tejidos renovables de aquellos de crecimiento continuo que generan líneas o capas donde almacenan la información mientras son formados. Información tomada de Tieszen et al. (1983) y Dalerum y Angerbjorn (2005). 


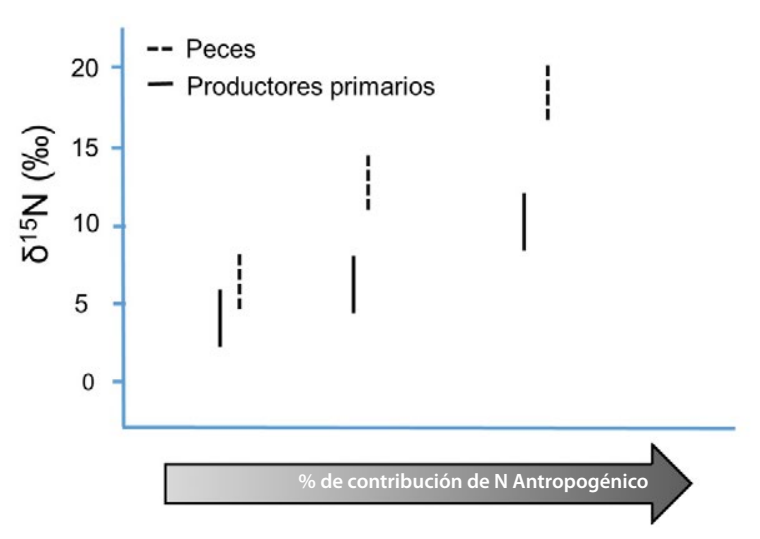

Figura 3. Rango de valores de $\delta^{15} \mathrm{~N}$ en productores primarios y en peces en relación al porcentaje de contribución por aguas residuales a la carga total de nitrógeno en sistemas costeros. Los datos fueron tomados de McClelland et al. (1997), Cole et al. (2004) y Schlacher et al. (2005).

Comúnmente, estos valores de fraccionamiento trófico son seleccionados en base a meta-análisis disponibles que resumen la información recabada tanto a nivel observacional como experimental (Post, 2002; McCutchan, et al., 2003; Vanderklift y Ponsard, 2003; Caut, et al., 2009).

Una ventaja significativa sobre los métodos tradicionales para el análisis de dieta radica en que diferentes tipos de tejidos tienen distintas tasas de recambio celular $\mathrm{y}$, por lo tanto, cada tejido brinda información de la dieta sobre una escala temporal específica. Por ejemplo, en el caso de las proteínas del plasma los isótopos estables dan información sobre la dieta en el rango de días y semanas, mientras que en el tejido muscular proveen información en la escala de meses (Dalerum y Angerbjorn, 2005; Tabla 1). En este sentido, se pueden realizar inferencias acerca de los patrones de movimiento y sitio de alimentación en diferentes rangos temporales comparando valores isotópicos en tejidos con diferente tasa de recambio. Fry et al. (2003) estudiaron los patrones de movimiento en camarones comparando los valores isotópicos en el músculo de la cola, que integra información de la dieta en semanas antes del muestreo, con el hepatopáncreas, que posee una tasa de recambio más rápida y proporciona información de la dieta diaria.

\section{Indicadores de impacto humano}

Los valores de $\delta^{15} \mathrm{~N}$ han sido utilizados para detectar nitrógeno de origen humano en ambientes costeros mediante el análisis de plantas y su biomagnificación en animales (McClelland, et al., 1997; Costanzo, et al., 2005). Esta práctica se basa en que los valores de $\delta^{15} \mathrm{~N}$ antropogénico originado por la descarga de aguas residuales se caracterizan por ser más positivos en comparación con el nitrógeno natural en ambientes marinos o de agua dulce. Esta diferencia isotópica se explica como consecuencia de una pérdida mayor de ${ }^{14} \mathrm{~N}$ durante la volatilización del amonio y la desnitrificación que ocurren en las plantas de tratamiento de aguas residuales de origen humano y que llevan a valores de $\delta^{15} \mathrm{~N}$ en un rango entre +10 a $+20 \%$ (McClelland, et al., 1997; Kendall, 1998). A su vez, este rango de variación de $\delta^{15} \mathrm{~N}$ es mayor al que presentan materiales como fertilizantes nitrogenados con bajos valores (rango entre +3 y $-3 \%$ ) que reflejan su fuente de $\mathrm{N}$ atmosférica (Kendall, 1998).

En este contexto, el enriquecimiento en ambientes costeros con ${ }^{15} \mathrm{~N}$ y el consecuente aumento de los valores de $\delta^{15} \mathrm{~N}$ producido por la contribución de nitrógeno de origen humano ha sido propuesto como indicador de eutrofización que permite detectar de forma temprana estos procesos en organismos bentónicos (Carlier, et al., 2008). Es esperable un patrón decreciente en los registros de $\delta^{15} \mathrm{~N}$, desde las zonas costeras con actividad humana hacia las zonas oceánicas, reflejo de la dilución de los nutrientes (Costanzo, et al., 2005; Vizzini y Mazzola, 2006). Sin embargo, se ha detectado en aguas residuales sin tratamiento bajos valores de $\delta^{15} \mathrm{~N}$ debido a un menor enriquecimiento de ${ }^{15} \mathrm{~N}$ por volatilización del amonio (Gaston y Suthers, 2004), lo que dificulta su utilidad como indicador de impacto antrópico si se usa aislado de otras herramientas complementarias como los nutrientes. Varios estudios han demostrado la influencia de la actividad humana y su impacto en la estructura ecosistémica mediante la evaluación de la composición isotópica en fauna y flora (McClelland, et al., 1997; Schlacher, et al., 2005; Figura 3). En este sentido, como indicadores de contaminación por nitrógeno se han utilizado con éxito plantas macrófitas (Cole, et al., 2004), peces juveniles (Schlacher, et al., 2005) y también invertebrados bentónicos (Riera, et al., 2000).

\section{Casos de estudio}

\section{Caracterización del origen de la materia orgánica en el Río de la Plata}

El Río de la Plata (RdlP; $34^{\circ} 10-36^{\circ} 20^{\prime} \mathrm{S}, 55^{\circ} 00^{\prime}-58^{\circ} 30^{\prime} \mathrm{W}$ ) es un cuerpo de agua con forma tipo embudo de $320 \mathrm{~km}$ de longitud y un ancho que varía entre los $2 \mathrm{~km}$ en su naciente y $230 \mathrm{~km}$ en su boca, configurando una superficie de $38.000 \mathrm{~km}^{2}$ (Giberto, et al., 2004). Debido a sus características hidrográficas el estuario puede dividirse en tres secciones: 1) estuario interno con profundidades menores a $5 \mathrm{~m}, 2$ ) estuario medio con profundidades menores a $10 \mathrm{~m}, \mathrm{y} 3$ ) el estuario externo de entre 10 y $20 \mathrm{~m}$ de profundidad (Nagy, et al., 2002). La determinación de las principales fuentes de materia orgánica (terrestre vs. marina) que se deposita a lo largo del gradiente salino en el RdlP se traduce en la composición isotópica de la matriz sedimentaria. Esta matriz está influida en gran medida por las grandes lluvias asociadas a los eventos «El Niño», que afectan la distribución espacial de las fuentes de material orgánico en el estuario. Esto implica cambios en las mediciones de $\delta^{13} \mathrm{C}$ y la relación $\mathrm{C} / \mathrm{N}$ en sedimentos superficiales y en testigos de sedimentos que fueron datados mediante análisis de radionucleidos $\left({ }^{210} \mathrm{~Pb}\right.$ and $\left.{ }^{137} \mathrm{Cs}\right)$.

Para analizar la distribución espacial de los tipos de materia orgánica se colectaron sedimentos superficiales en un total de 26 estaciones que contemplaron la zona interna, media y externa del RdlP. Para el análisis temporal y su relación con los eventos «El Niño» se colectaron seis testigos de sedimentos utilizando un tubo de PVC de $10.3 \mathrm{~cm}$ de diámetro interno y una longitud que varió entre los 51 y los $115 \mathrm{~cm}$; dos testigos se colectaron en la zona interna del estuario adyacente a la descarga del Río Uruguay y Paraná y otros cuatro testigos fueron tomados en la zona externa del RdlP (ver Bergamino, et al., 2017). Una vez en el laboratorio, para los análisis isotó- 
picos cada testigo fue seccionado longitudinalmente cada 2 $\mathrm{cm}$ hasta los $20 \mathrm{~cm}$ del tubo de sedimento, cada $3 \mathrm{~cm}$ entre los 20 y $50 \mathrm{~cm}$ y finalmente cada $5 \mathrm{~cm}$ (Bergamino, et al., 2017).

La distribución espacial de los valores de $\delta^{13} \mathrm{C}$ en los sedimentos superficiales mostraron un aumento progresivo desde el estuario interno, con valores que variaron entre -25 a $-26 \%$, hacia el estuario medio y externo donde se observaron valores entre -20 y $-22 \%$. A su vez, los resultados isotópicos de los testigos localizados en la zona interna del estuario adyacente a la descarga del Río Uruguay mostraron tendencias claras con una disminución en los valores de $\delta^{13} \mathrm{C}$ desde los sedimentos más antiguos hacia los recientes y con un rango de variación entre - 28 y -22\%o (Bergamino, et al., 2017). Además, se registraron cambios en las tendencias con disminuciones abruptas de $\delta^{13} \mathrm{C}$ durante años de eventos «El Niño», en especial durante los años 1982/1983 y 1997/1998. Los valores de $\mathrm{C} / \mathrm{N}$ estuvieron siempre por encima de 9 para estos testigos adyacentes a la descarga de los ríos Paraná y Uruguay. Los sedimentos de los testigos ubicados en la zona externa del RdlP mostraron un rango de variación de $\delta^{13} \mathrm{C}$ más positivo, que varió entre -19 y $-10 \%$.

Estos resultados de cambios isotópicos a lo largo del estuario sugieren una diferenciación espacial en términos del tipo de materia orgánica que afectan al RdlP. En este sentido, los valores más negativos de $\delta^{13} \mathrm{C}$ hacia la zona interna del estuario se pueden asociar a una influencia mayor de material orgánico de origen continental, por ejemplo, plantas vasculares locales y fitoplancton dulceacuícola, cuyos valores de $\delta^{13} \mathrm{C}$ varían entre -29 y $-26 \%$, en consonancia con los observados en el estuario interno (testigos y sedimento superficial) (Goñi, et al., 2003). Este patrón isotópico contrasta con los valores registrados en los testigos y sedimentos superficiales en la zona media y externa, reflejando un cambio geográfico en las fuentes de materia orgánica que se deposita en el estuario. Los mayores valores isotópicos de $\delta^{13} \mathrm{C}$ observados en los sedimentos hacia la zona externa indican una importante contribución del fitoplancton marino con valores característicos entre -21 y -18\%o (Lamb, et al., 2006). Los sitios externos del estuario posiblemente presenten una mayor influencia de procesos oceanográficos sin estar afectados significativamente por una descarga de agua dulce que favorezca la disponibilidad de detritos de origen terrestres y con bajos valores de $\delta^{13} \mathrm{C}$.

El cambio en las fuentes de materia orgánica a lo largo del RdlP parece estar asociado al gradiente salino. Asimismo, la tendencia de $\delta^{13} \mathrm{C}$ a disminuir durante eventos El Niño sugiere que los patrones de descarga de agua dulce asociados a estos eventos promueven una mayor influencia de material terrestre originado en las zonas adyacentes al estuario interno. En este sentido, los patrones hidrológicos junto con la vegetación dominante pueden determinar los tipos de materia orgánica que se deposita y queda disponible en los sistemas estuarinos. En este caso, se evidencia que la composición isotópica de $\delta^{13} \mathrm{C}$ y la relación $\mathrm{C} / \mathrm{N}$ son indicadores útiles para identificar cambios en las fuentes de material orgánico en sistemas costeros y realizar reconstrucciones paleoambientales.

\section{Cambios en las relaciones alimentarias en playas arenosas}

Los valores isotópicos de los ejes $\delta^{13} \mathrm{C}$ y $\delta^{15} \mathrm{~N}$ integran información acerca de los recursos y del tipo de hábitat que los animales utilizan y, por lo tanto, son útiles para definir el espacio del nicho ecológico (Martínez del Río, et al., 2009).
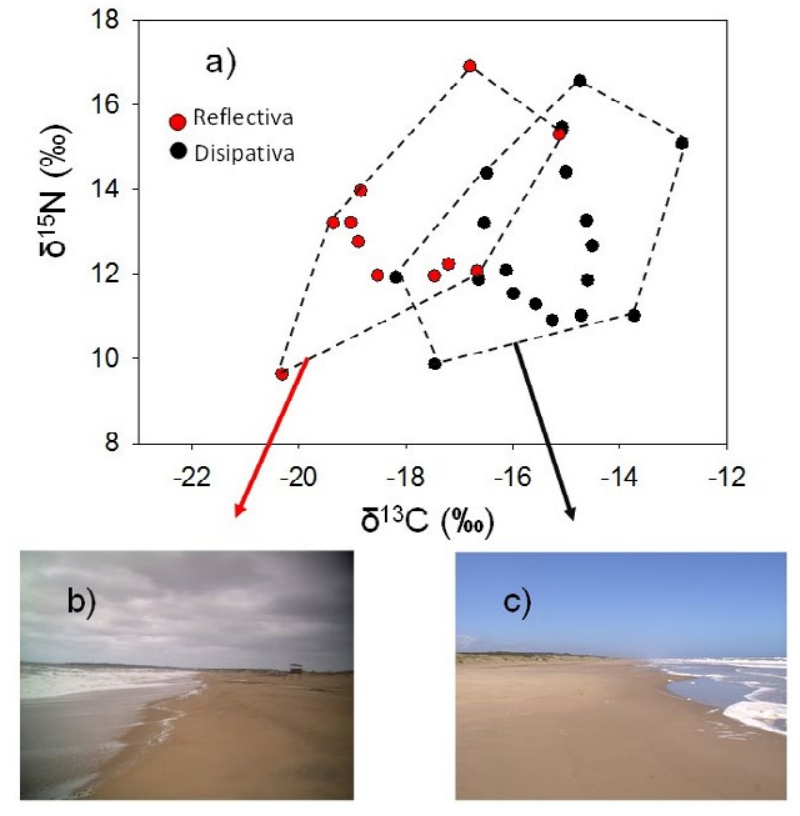

Figura 4. a) Representación del $\delta$-espacio para la macrofauna bentónica residente en una playa con morfodinámica reflectiva (b: Arachania) y disipativa (c: Barra del Chuy). Las líneas punteadas representan el polígono de $\delta^{13} \mathrm{C}$ y $\delta^{15} \mathrm{~N}$ que define el nicho trófico isotópico total para cada playa. Los datos fueron adaptados de Bergamino et al. $(2011,2016)$.

De esta manera, los valores de $\delta^{13} \mathrm{C}$ y $\delta^{15} \mathrm{~N}$ se distribuyen en un $\delta$-espacio que ha sido formalizado para investigar el nicho trófico isotópico de especies o de una comunidad, analizando el volumen de espacio isotópico que cubre la dispersión de los valores isotópicos (Newsome, et al., 2007; Figura 4). Existen estudios que promueven el uso de la distribución de los valores isotópicos en el $\delta$-espacio, para evaluar cuantitativamente las diferencias en la estructura trófica en diferentes hábitats o tiempos (Layman, et al., 2007; Schmidt, et al., 2007).

Se analizaron los cambios en los nichos tróficos isotópicos de las especies de la macrofauna bentónica de dos playas ubicadas en la costa atlántica de Uruguay en el departamento de Rocha caracterizadas por una morfodinámica contrastante (Bergamino, et al., 2016). Específicamente, para este estudio se consideró la fauna residente en una playa disipativa (Barra del Chuy: grano fino y pendiente suave) y otra reflectiva (Arachania: grano grueso y pendiente pronunciada). En las playas disipativas localizadas en la costa atlántica de Uruguay y sur de Brasil es frecuente observar grandes acumulaciones de microalgas dominandas por la diatomea de rompiente Asterionellopsis glacialis (Odebrecht, et al., 2014), recientemente descrita como Asterionellopsis guyunusae (Kaczmarska, et al., 2014). Sin embargo, la importancia de esta diatomea en la estructura trófica de playas arenosas ha sido poco evaluada en Uruguay.

La colecta de la macrofauna se realizó simultáneamente en ambas playas en forma estacional (primavera: noviembre 2008, verano: febrero 2009, otoño: abril 2009, e invierno: julio 2009). Además, se colectaron muestras de material orgánico particulado (MOP), y muestras monoespecíficas de la dia- 
tomea A. guyunusae durante un evento de acumulación en la playa disipativa de Barra del Chuy, con un filtro de fibra de vidrio Whatman GF/C. Las muestras de A. guyunusae no fueron colectadas en la playa reflectiva, ya que acumulaciones de esta especie solo ocurren en la playa disipativa (Bergamino, et al., 2016).

Los valores isotópicos de la diatomea A. guyunusae mostraron un rango de variación de entre -17 y $-16 \%$ para $\delta^{13} \mathrm{C}$ y desde 7 a $9 \%$ para $\delta^{15} \mathrm{~N}$ (Bergamino, et al., 2016). Estos valores isotópicos fueron significativamente superiores a los valores encontrados para el MOP con un rango de variación de entre -22 a $-20 \%$ en la playa disipativa y de entre -20 a $-19 \%$ en la playa reflectiva. Las diferencias en la red trófica muestran un claro cambio direccional en el espacio del nicho isotópico para las especies que habitan en ambas playas, y se constata una utilización de recursos enriquecidos en ${ }^{13} \mathrm{C}$ en la playa disipativa (Figura 4). Las diferencias derivaron principalmente de los cambios direccionales de los vectores asociados a las especies de invertebrados Atlantorchestoidea brasiliensis, Emerita brasiliensis y Excirolana sp.

En este contexto, los mayores valores isotópicos de la diatomea A. guyunusae en comparación con MOP marino de ambas playas y las diferencias en el espacio isotópico de distintas especies entre las playas estarían explicadas por una mayor asimilación de las diatomeas que habitan la playa disipativa. La mayor concentración de $\mathrm{N}$ en estas diatomeas, que aumenta su calidad nutricional, puede ser un factor de cambio de los nichos tróficos isotópicos entre las playas arenosas con morfodinámica contrastante. Así, las playas disipativas con presencia de diatomeas provenientes de la zona de rompiente podrían sostener altas abundancias de filtradores, debido al mayor contenido proteico de esta especie en relación al MOP disponible en las playas reflectivas.

\section{Materia orgánica antropogénica en los sedimentos de la Bahía de Montevideo}

Se evaluó la variabilidad histórica natural y antrópica en la composición de la materia orgánica depositada en la Bahía de Montevideo (BM) a través de mediciones de isótopos estables en un testigo de sedimentos. Este sistema se encuentra severamente degradado por diferentes presiones antrópicas, incluyendo la descarga de aguas residuales y naturales asociadas con eventos El Niño (Danulat, et al., 2002; Muniz, et al., 2011; García- Rodríguez, et al., 2014). En la actualidad la zona oeste de Montevideo carece de saneamiento, por lo que el aumento de la población en su cuenca implicó un incremento en el volumen de los efluentes domésticos y, en consecuencia, de su contenido orgánico. Con respecto a la variabilidad climática, para la cuenca baja del RdlP se observó un aumento de las descargas del Paraná hacia fines del siglo XX, en particular a partir de la década de 1950 (López Laborde y Nagy, 1999; Jaime y Menéndez, 2002). Adicionalmente, los eventos «El Niño» comenzaron a ser más intensos desde ese entonces (McPhaden, et al., 2006).

Para establecer las diferentes magnitudes de esta variabilidad natural y antrópica del sistema se analizaron los isótopos estables $\delta^{13} \mathrm{C}, \delta^{15} \mathrm{~N}$ y la relación $\mathrm{C} / \mathrm{N}$ en los sedimentos de un testigo de sedimento colectado en la zona interna de la BM en mayo de 2010. El testigo presentó $6,3 \mathrm{~cm}$ de diámetro interno, $149 \mathrm{~cm}$ de largo y se seccionó en estratos de $1 \mathrm{~cm}$ de espesor (Bueno, et al., 2016).
La tasa de sedimentación $\left(0,53 \mathrm{~cm} \cdot \mathrm{año}^{-1}\right)$ (Bueno, et al., 2016) permitió determinar que desde la base del testigo (correspondiente al año 1860) y hasta fines de la década de 1940 la materia orgánica presentó un origen mayormente marino $\left(\delta^{13} \mathrm{C} \approx-21 \%\right.$ y $\left.\mathrm{C} / \mathrm{N} \approx 10\right)$. Luego, a partir de la década de 1950 aproximadamente, se observó un cambio en los aportes al sistema, con una materia orgánica de origen mixto $\left(\delta^{13} \mathrm{C} \approx-24,5 \%\right.$ y $\left.\mathrm{C} / \mathrm{N} \approx 16\right)$. A partir de esa década se observa un cambio en la señal isotópica de $\delta^{15} \mathrm{~N}$ (promedio de $\delta^{15} \mathrm{~N}=6,81 \pm 0,61 \%$ en la base y $5,10 \pm 0,42 \%$ en la superficie) por mayor aporte de origen terrestre y efluentes domésticos sin tratamiento. Diversos autores señalan que los efluentes de origen doméstico presentan una baja firma isotópica de $\delta^{15} \mathrm{~N}$ con respecto a los que tienen algún tratamiento (Sampaio, et al., 2010).

En todas las variables analizadas $\left(\delta^{13} \mathrm{C}, \delta^{15} \mathrm{~N}\right.$ y C/N $)$ se observó un cambio en las tendencias entre fines de la década de 1940 y comienzos de la década de 1950. Estos resultados podrían explicarse por un cambio en el uso de la cuenca de drenaje, por el incremento de la población e industrias $y$, en consecuencia, por el aumento del vertido de efluentes domésticos e industriales. Con respecto a la variabilidad climática, se comparó los eventos «El Niño» fuertes y muy fuertes (1950-2010), y estos no se correspondieron con los cambios observados en la relación C/N. La historia de contaminación de metales de la BM, relevada mediante el índice de Contaminación de Sedimentos (SPI), se relacionó muy bien con la tendencia de la relación C/N (Bueno, 2016), con una correlación de 0,86 , lo que refuerza la idea de un origen antrópico del carbono orgánico en los sedimentos históricos de la BM. En este sentido, si bien existe una variabilidad natural ya establecida (López Laborde y Nagy, 1999; McPhaden, et al., 2006), esta señal se estaría perdiendo debido a la presión antrópica existente sobre la BM (Bueno, 2016).

\section{Conclusiones y perspectivas}

La variación isotópica en $\delta^{13} \mathrm{C}$ de la materia orgánica depositada a lo largo del estuario del RdlP da cuenta de un cambio en las fuentes disponibles para los consumidores primarios, incluyendo plantas vasculares terrestres y fitoplancton de origen marino (Bergamino, et al., 2017). La utilización de la relación $\mathrm{C} / \mathrm{N}$ en los sedimentos puede ser difícil ya que se han documentado valores por debajo de 20 no solamente para material marino, sino también para material de origen terrestre (Zhao, et al., 2015).

Los valores isotópicos de las fuentes de material orgánico pueden variar temporal y espacialmente y estas variaciones pueden afectar los modelos usados y las interpretaciones. Pequeñas diferencias de \%o en $\delta^{13} \mathrm{C}$ pueden alterar significativamente la interpretación de los resultados (Vander Zanden y Rasmussen, 2001). A su vez, la comparación de la variación isotópica entre consumidores es susceptible a la variación intrínseca de las presas y, por lo tanto, debe incluirse esta variación en el análisis y en los respectivos diseños de muestreo.

Este trabajo intenta mostrar la valiosa información que pueden registrar los isótopos estables sobre la dinámica en las fuentes de carbono y las formas en que interactúan los animales con su ambiente. La aplicación de isótopos estables debe ser vista como una herramienta complementaria que compensa las debilidades de otras técnicas para la caracterización de la materia orgánica y sus vías de transferencia en 


\begin{tabular}{lll}
\hline Recurso & Categoría & Dirección electrónica \\
\hline ISOGEOCHEM & Foro & http://isogeochem.wikispaces.com/ \\
Isonumbers & Base de datos & http://isonumbers.weizmann.ac.il/resources.aspx \\
USGS & Fundamentos & https://wwwrcamnl.wr.usgs.gov/isoig/res/funda.html \\
Isoscapes & Análisis de datos & https://sites.google.com/site/westlabgroup/isoscapes-home \\
SINLAB & Preparación de muestras & $\begin{array}{l}\text { http://www.unb.ca/research/institutes/cri/sinlab/ } \\
\text { samplecollectionpreparation.html }\end{array}$ \\
GNIR & Base de datos & http://www-naweb.iaea.org/napc/ih/IHS_resources_gnir.html \\
CATNAS & Laboratorio en Uruguay & http://www.fagro.edu.uy/ catnas/ \\
\hline
\end{tabular}

Tabla 2. Resumen de algunos recursos electrónicos para el análisis de isótopos estables: base de datos, foros de discusión y métodos de preparación de muestras.

la red trófica. Por ejemplo, en el contexto de redes tróficas, si bien la información isotópica en animales carece de resolución taxonómica, brinda información de la dieta integrada en el tiempo. Esta aproximación trófica usada en conjunto con otros tipos de análisis (por ejemplo, contenido estomacal, heces) y con un conocimiento previo del sistema permitirá un entendimiento completo sobre la estructura y los flujos de energía que ocurren en el sistema de estudio. En un contexto de reconstrucción paleoambiental el análisis de polen y de las diatomeas contenidas en los registros de sedimentos permite apoyar interpretaciones acerca de cambios en la vegetación pasada (Byrne et al., 2001).

El conocimiento sobre los procesos ambientales y fisiológicos que transforman los valores isotópicos y generan fraccionamiento es importante para alcanzar interpretaciones robustas. Por lo tanto, la generalización de factores de fraccionamientos debe hacerse con cuidado ya que puede dar lugar a representaciones erróneas de los modelos isotópicos que se generen, con un mayor error que valores estimados mediante experimentos (Caut, et al., 2008). La generación de datos isotópicos históricos para caracterizar el estado inicial de los ecosistemas resulta un desafío importante para evaluar cambios a largo plazo en la estructura ecosistémica (Vander Zanden, et al., 2003). En este sentido, la utilización de muestras almacenadas en museos puede ser estudiada isotópicamente aplicando correcciones para los efectos químicos generados por los preservantes y es un camino alentador para reconstruir redes tróficas pasadas (González Bergonzio, et al., 2015). Esta aproximación permitiría evaluar el impacto de especies introducidas así como el efecto que ha tenido en los ecosistemas la urbanización en zonas costeras.

La técnica de medición isotópica es una herramienta transversal a varias disciplinas y su aplicación en investigaciones ecológicas y geoambientales es una evidente oportunidad. Actualmente, existen numerosos recursos disponibles para investigadores que permiten aprender y profundizar el conocimiento de las formas de aplicación de isótopos estables en ciencias ambientales, entre otros, los foros de consultas y las bases de datos (Tabla 2).

Este trabajo puede servir como guía básica para que futuros investigadores puedan evaluar contribuciones científicas mediante la aplicación de isótopos estables y diseñar protocolos consistentes con esta técnica.

\section{Reconocimientos}

Los autores agradecen a la Agencia Nacional de Investigación e Innovación (ANII), por el apoyo financiero mediante el Sistema Nacional de Investigadores (L.B. y F.G.R.) y el programa de Becas de Posgrado (A.T. y C.B.). Los autores agradecen también al PEDECIBA, Intendencia de Montevideo y Administración Nacional de Puertos por el financiamiento. L.B agradece además al programa CSIC «Contratación de Científicos Provenientes del Exterior»y al programa CSIC I+D 2016 por el financiamiento.

\section{Referencias}

Bergamino, L., Lercari, D. y Defeo, O., 2011. Food web structure of sandy beaches: temporal and spatial variation using stable isotope analysis. En: Estuarine, Coastal and Shelf Science, 91, pp.536-543.

Bergamino, L., Martínez, A., Han, E., Lercari, D., y Defeo, O., 2016. Trophic niche shifts driven by phytoplankton in sandy beach ecosystems. En: Estuarine, Coastal and Shelf Science, 180, pp.33-40.

Bergamino, L., Schuerch, M., Tudurí, A., Carretero, S., y Garcia-Rodriguez, F., 2017. Linking patterns of freshwater discharge and sources of organic matter within the Río de la Plata estuary and adjacent marshes. En: Marine and Freshwater Research, en prensa.

Berke, M.A., Johnson, T.C., Werne J.P., Grice, K., Schouten, S., y Sinninghe Damsté J.S., 2012. Molecular records of climate variability and vegetation response since the Late Pleistocene in the Lake Victoria basin, East Africa. En: Quaternary Science Reviews, 55(8), pp.59-74.

Bueno, C., 2016. Isótopos estables de C y N, y la relación Corg/ Norg como indicadores del origen de la materia orgánica en los sedimentos de la zona costera de Montevideo: un análisis histórico y actual. Montevideo: Facultad de Ciencias. (Tesis de Maestría).

Bueno, C., Brugnoli, E., Figueira, R.C.L., Muniz, P., Ferreira, P.A.L. y García Rodríguez, F., 2016. Historical economic and environmental policies influencing trace metal inputs in Montevideo Bay, Río de la Plata. En: Marine Pollution Bulletin, 113, pp.141-146. 
Byrne, R., Ingram, L.B., Starratt, S. y Malamud-Roam, F., 2001. Carbon-isotope, diatom, and pollen evidence for late Holocene salinity change in a brackish marsh in the San Francisco Estuary. En: Quaternary Research, 55, pp.66-76.

Carlier, A., Riera, P., Amouroux, J.-M., Bodiou, J.-Y., Desmalades, M. y Grémare, A., 2008. Foodweb structure of two Mediterranean lagoons under varying degree of eutrophi-cation. En: Journal of Sea Research, 60, pp.264-275.

Caut, S., Angulo, E. y Courchamp, F., 2008. Caution on isotopic model use for analyses of consumer diet. En: Canadian Journal of Zoology, 86, pp.438-445.

Caut, S., Angulo, E. y Courchamp, F., 2009. Variation in discrimination factors $\left(\delta^{15} \mathrm{~N}\right.$ and $\left.\delta^{13} \mathrm{C}\right)$ : the effect of diet isotopic values and applications for diet reconstruction. En: Journal of Applied Ecology, 46, pp.443-453.

Cerling, T.E., Harris, J.M. y Leakey, M.G., 1999. Browsing and grazing in elephants: the isotope record of modern and fossil proboscideans. En: Oecologia, 120, pp.364-374.

Cole, M.L., Valiela, I., Kroeger; K.D., Tomasky; G.L., Cebrian, J., Wigand, C., McKinney, R.A., Grady S.P. y Carvalho da Silva, M.H., 2004. Assessment of $\delta^{15} \mathrm{~N}$ isotopic method to indicate anthropogenic eutrophication in aquatic ecosystems. En: Journal of Environmental Quality, 33, pp.124-132.

Costanzo, S.D., Udy, J., Longstaff, B. y Jones, A., 2005. Using nitrogen stable isotope ratios $\left(\delta^{15} \mathrm{~N}\right)$ of macroalgae to determine the effectiveness of sewage upgrades: changes in the extent of sewage plumes over four years in Moreton Bay, Australia. En: Marine Pollution Bulletin, 51, pp.212-217.

Craven, K.F., Edwards, R.J., y Flood, R.P., 2017. Source organic matter analysis of saltmarsh sediments using SIAR and its application in relative sea-level studies in regions of C4 plant invasion. En: Boreas, en prensa.

Dalerum, F. y Angerbjorn, A., 2005. Resolving temporal variation in vertebrate diets using naturally occuring stable isotopes. En: Oecologia, 144, pp.647-658.

Danulat, E., Muniz, P., García Alonso, J., y Yannicelli, B., 2002. First assessment of the highly contaminated harbour of Montevideo, Uruguay. En: Marine Pollution Bulletin, 44, pp.554-565.

DeNiro, M.J. y Epstein, S., 1978. Influence of diet on the distribution of car- bon isotopes in animals. En: Geochimica Et Cosmochimica Acta, 42, pp.495-506.

Finlay, J.C., Power, M.E. y Cabana, G., 1999. Effects of water velocity on algal carbon isotope ratios: implications for river food web studies. En: Limnology and Oceanography, 44, pp.1198-1203.

Franco-Trecu, V., Aurioles-Gamboa, D. y Inchausti, P., 2014. Individual trophic specialisation and niche segregation explain the contrasting population trends of two sympatric otariids. En: Marine Biology, 161, pp.609-618.

Fry, B., Baltz, D.M., Benfield, M., Fleeger, J., Gace, A., Haas, H. y Quiñones-Rivera, Z., 2003. Stable isotope indicators of movement and residency for brown shrimp (Farfantepenaeus aztecus) in coastal Louisiana marshscapes. En: Estuaries, 26, pp.82-97.

Gannes, L.Z., Martínez del Rio, C. y Koch, P., 1998. Natural abundance variations in stable isotopes and their potential uses in animal physiological ecology. En: Comparative Biochemistry and Physiology A - Molecular and Integrative Physiology, 119, pp.725-737.
García-Rodríguez, F., Brugnoli, E., Muniz, P., Venturini, N., Burone, L., Hutton, M. Rodríguez, M., Pita, A., Kandratavicius, N., Perez, L. y Verocai, J., 2014. Warm-phase ENSO events modulate the fluvial freshwater input and the trophic state of sediments in a large South American estuary. En: Marine and Freshwater Research, 65, pp.1-11.

Gaston, T.F. y Suthers, I.M., 2004. Spatial variation in d13C and $\mathrm{d} 15 \mathrm{~N}$ of liver, muscle and bone in a rocky reef planktivorous fish: the relative contribution of sewage. En: Journal of Experimental Marine Biology and Ecology, 304, pp.17-33.

Giberto, D. A., Bermec, C. A., Acha, E. M. y Mianzan, H., 2004. Large- scale spatial patterns of benthic assemblages in theSWAtlantic: the Rio de la Plata Estuary and adjacent shelf waters. En: Estuarine, Coastal and Shelf Science, 61, pp.1-13.

González Bergonzoni, I., Vidal, N., Wang, B., Ning, D., Liu, Z., Jeppesen, E. y Meerhoff, M., 2015. General validation of formalin-preserved fish samples in food web studies using stable isotopes. En: Methods in Ecology and Evolution, 6, pp.307-314.

Goñi, M.A., Teixeira, M.J. y Perkey, D.W., 2003. Sources and distribution of organic matter in a river-dominated estuary (Winyah Bay, SC, USA). En: Estuarine, Coastal and Shelf Science, 57, pp.1023-1048.

$\mathrm{Gu}$, B., Schelske, C.L. y Brenner, M., 1996. Relationship between sediment and plankton isotope ratios $\left(\delta^{13} \mathrm{C}\right.$ and $\left.\delta^{15} \mathrm{~N}\right)$ and primary productivity in Florida lakes. En: Canadian Journal of Fisheries and Aquatic Sciences, 53, pp.875-883.

Hoefs, J., 2009. Stable isotope geochemistry. 6ta. ed. Berlín: Springer-Verlag.

Jaime, P. y Menéndez, A.N., 2002. Informe análisis del régimen hidrológico de los ríos Paraná y Uruguay. Proyecto protección ambiental del Río de la Plata y su frente marítimo: prevención y control de la contaminación y restauración de hábitats. PNUD/GEF, RLA/99/G31. Buenos Aires: Instituto Nacional del Agua.

Kaczmarska, I., Mather, L., Luddington, I.A., Muise, F. y Ehrman, J.M., 2014. Cryptic diversity in a cosmopolitan diatom known as Asterionellopsis glacialis (Fragilariaceae): implications for ecology, biogeography and taxonomy. En: American Journal of Botany, 101, pp.267-286.

Kendall, C., 1998. Tracing nitrogen sources and cycling in catchments. En: Kendall, C., McDonnell, J.J., eds., 1998. Isotope tracers in catchment hydrology. Amsterdam: Elsevier. pp.519-576.

Lamb, A.L., Wilson, G.P. y Leng, M.J., 2006. A review of coastal palaeoclimate and relative sea-level reconstructions using $\delta^{13} \mathrm{C}$ and $\mathrm{C} / \mathrm{N}$ ratios in organic material. En: Earth Science Reviews, 75, pp.29-57.

Layman, C.A, Arrington, D.A., Montaña C.G. y Post, D.M., 2007. Can stable isotope ratios provide for community-wide measures of trophic structure? En: Ecology, 88, pp.42-48.

López Laborde, J.L. y Nagy, G.J., 1999. Hydrography and sediment transport characteristics of the Río de la Plata: a review. En: Perillo, M. E., Piccolo, M. C. y PinoQuivira, M., eds., 1999. Estuaries of South America, their geomorphology and dynamics. Berlín: Springer. pp.133-157.

Martínez del Río, C., Wolf, N., Carleton, S.A., y Gannes, L.Z., 2009. Isotopic ecology ten years after a call for more laboratory experiments. En: Biological Reviews, 84, pp.91-111. 
McPhaden, M. J., Zebiak, S.E. y Glantz, M.H., 2006. ENSO as an integrating concept in Earth science. En: Science, 314, pp.1740-1745.

McClelland, J.W., Valiela, I. y Michener, R.H., 1997. Nitrogenstable isotope signatures in estuarine food webs: a record of increasing urbanization in coastal watersheds. En: Limnology and Oceanography, 42, pp.930-937.

McCutchan, J.H., Lewis, W.M., Kendall, C. y McGrath, C.C., 2003. Variation in trophic shift for stable isotope ratios of carbon, nitrogen, and sulfur. En: Oikos, 102, pp.378- 390.

Meyers, P.A., 1994. Preservation of elemental and isotopic source identification of sedimentary organic matter. En: Chemical Geology, 114, pp.289-302.

Michener, R.H., y Schell, D.M., 1994. Stable isotope ratios as tracers in marine aquatic food webs. En: Michener, R.H., Lajtha, K., eds., 1994. Stable isotopes in ecology and environmental science. 2 da ed. Malden: Blackwell. pp.138-157.

Muniz, P., Venturini, N., Hutton, M., Kandratavicius, N., Pita, A., Brugnoli, E., Burone, L. y García-Rodríguez, F., 2011. Ecosystem health of Montevideo coastal zone: A multi approach using some different benthic indicators to improve a ten-year-ago assessment. En: Journal of Sea Research, 65, pp.38-50.

Nagy, G. J., Gómez-Erache, M., López, C. H. y Perdomo, A. C., 2002. Distribution patterns of nutrients and symptoms of eutrophication in the Rio de la Plata estuary. En: Hydrobiologia, 475/476, pp.125-139.

Newsome, S.D., Martínez del Rio, C., Bearhop, S. y Phillips, D.L., 2007. A niche for isotopic ecology. En: Frontiers in Ecology and the Environment, 5, pp.429-36.

Nier, A.O., 1947. A mass spectrometer for isotopes and gas analysis. En: Review of Scientific Instruments, 18, pp.398-411.

Odebrecht, C., Du Preez, D.R., Abreu, P.C. y Campbell, E.E., 2014. Surf zone diatoms: a review of the drivers, patterns and role in sandy beaches food chains. En: Estuarine, Coastal and Shelf Science, 150, pp.24-35.

O'Leary, M.H., 1981. Carbon isotope fractionation in plants. En: Phytochemistry, 20, pp. 553-567.

Perez, L., García-Rodríguez, F. y Hanebuth, T.J.J., 2017. Paleosalinity changes in the Río de la Plata estuary and on the adjacent Uruguayan continental shelf over the past 1200 years: an approach using diatoms as a proxy. En: Weckström, K., Saunders, K., Gell, P. y Skilbeck, G., eds., 2017. Applications of paleoenvironmental techniques in estuarine studies springer Netherlands. Berlín: Springer. ISBN 978-94-024-0988-8.

Phillips, D.L., Inger, R., Bearhop, S., Jackson, A.L., Moore, J.W., Parnell, A.C., Semmens, B.X., y Ward, E.J., 2014. Best practices for use of stable isotope mixing models in food web studies. En: Canadian Journal of Zoology, 92, pp.823-835.

Post, D.M., 2002. Using stable isotopes to estimate trophic position: models, methods, and assumptions. En: Ecology, 83, pp.703-718.

Riera, P., Stal, L. y Niewenhuize, J., 2000. Heavy $\delta^{15} \mathrm{~N}$ in intertidal benthic algae and invertebrates in the Scheldt Estuary (the Netherlands): Effects of river nitrogen inputs. En: Estuarine, Coastal and Shelf Science, 51, pp.365-372.
Rodríguez-Graña, L., Calliari, C., Conde, D., Sellanes, J. y Urrutia, R., 2008. Food web of a SW Atlantic shallow coastal lagoon: spatial environmental variability does not impose substantial changes in the trophic structure. En: Marine Ecology Progress Series, 362, pp.69-83.

Rosenbauer, R.J., Swarzenski, P.W., Kendall, C., Orem, W.H., Hostettler, F.D., Rollog, M.E., 2009. A carbon, nitrogen, and sulfur elemental and isotopic study in dated sediment cores from the Louisiana Shelf. En: Geo-Marine Letters, 29, pp.415-429.

Rubenstein, D.R., y Hobson, K.A., 2004. From birds to butterflies: animal movement patterns and stable isotopes. En: Trends in Ecology and Evolution, 19, pp.256-263.

Sampaio, L., Freitas, R., Máguas, C., Rodrigues, A. y Quintino, V., 2010. Coastal sediments under the influence of multiple organic enrichment sources: An evaluation using carbon and nitrogen stable isotopes. En: Marine Pollution Bulletin, 60, pp.272-282.

Schmidt, S.N., Olden, J.D., Solomon, C.T. y Vander Zanden, M.J., 2007. Quantitative approaches to the analysis of stable isotope food web data. En: Ecology, 88, pp.2793-2802.

Schlacher, T.A., Liddell, B., y Gaston, T.F. y SchlacherHoenlinger, M., 2005. Fish track wastewater pollution to estuaries. En: Oecologia, 144, pp.570-584.

Sulzman, E.W., 2007. Stable isotope chemistry and measurement: a primer. En: Michener, R. y Lajtha, K., eds., 2007. Stable isotopes in ecology and environmental science. 2da ed. Boston: Blackwell Publishing. ISBN: 9781405126809.

Teece, M.A., y Fogel, M.L., 2004. Preparation of ecological and biochemical samples for isotope analysis. En: De Groot, P.A., ed., 2004. Handbook of stable isotope analytical techniques. Vol. 1. Amsterdam: Elsevier. pp.177-202.

Tieszen, L.L., Boutton, T.W., Tesdahl, K.G. y Slade, N.A., 1983. Fractionation and turnover of stable carbon isotopes in animal tissues: implications for ?13C analysis of diet. En: Oecologia, 57, pp.32-37.

Vander Zanden, M.J. y Rasmussen, J.B., 2001. Variation in $\delta^{15} \mathrm{~N}$ and $\delta^{13} \mathrm{C}$ trophic fractionation: implications for aquatic food web studies. En: Limnology and Oceanography, 46, pp.2061-2066.

Vander Zanden, M.J., Chandra, S., Allen, B.C., Reuter, J.E. y Goldman, C.R., 2003. Historical food web structure and restoration of native aquatic communities in the Lake Tahoe (California-Nevada) basin. En: Ecosystems, 6, pp.274-288.

Vanderklift, M.A. y Ponsard, S., 2003. Sources of variation in consumer-diet $\delta 15 \mathrm{~N}$ enrichment: a meta analysis. En: Oecologia, 136, pp.169-182.

Vizzini, S. y Mazzola, A., 2006. The effects of anthropogenic organic matter inputs on stable carbon and nitrogen isotopes in organisms from different trophic levels in a southern Mediterranean coastal area. En: Science of the Total Environment, 368, pp.723-731.

West, J. B., Bowen, G.J., Cerling, T.E. y Ehleringer, J.R., 2006. Stable isotopes as one of natures ecological recorders. En: Trends in Ecology and Evolution, 21, pp.408-414.

Zhao, Y., Wu, F., Fang, X. y Yang, Y., 2015. Topsoil C/N ratios in the Qilian Mountains area: Implications for the use of subaqueous sediment $\mathrm{C} / \mathrm{N}$ ratios in paleoenvironmental reconstructions to indicate organic sources. En: Palaeogeography, Palaeoclimatology, Palaeoecology, 426, pp.1-9. 